\title{
Audio-visual predictive processing in perception of humans and robots
}

2

\author{
Busra Sarigul $^{1,2}$, Burcu A. Urgen ${ }^{3,4,5}$ \\ ${ }^{1}$ Artificial Intelligence Program, Ankara University \\ ${ }^{2}$ Department of Psychology, Nuh Naci Yazgan University \\ ${ }^{3}$ Department of Psychology, Bilkent University \\ ${ }^{4}$ Interdisciplinary Neuroscience Program, Bilkent University \\ ${ }^{5}$ Aysel Sabuncu Brain Research Center and National Magnetic Resonance Imaging Research \\ Center (UMRAM)
}

\begin{abstract}
Recent work in cognitive science suggests that our expectations affect visual perception. With the rise of artificial agents in human life in the last few decades, one important question is whether our expectations about non-human agents such as humanoid robots affect how we perceive them. In the present study, we addressed this question in an audio-visual context. Participants reported whether a voice embedded in a noise belonged to a human or a robot. Prior to this judgment, they were presented with a human or a robot image that served as a cue and allowed them to form an expectation about the category of the voice that would follow. This cue was either congruent or incongruent with the category of the voice. Our results show that participants were faster and more accurate when the auditory target was preceded by a congruent cue than an incongruent cue. This was true regardless of the human-likeness of the robot. Overall, these results suggest that our expectations affect how we perceive non-human agents and shed light on future work in robot design.
\end{abstract}

Keywords: prediction, expectation violation, human-robot interaction, audio-visual mismatch 


\section{Introduction}

Advances in artificial intelligence in the last few decades have introduced us to humanoid

27 robots that we encounter everywhere ranging from classrooms to airports to shopping malls to

28 hospitals. While their presence in our daily lives has brought a lot of excitement, how humans

29 perceive and interact with them has become an important research topic in cognitive science. Do

30 we perceive them differently from the way we perceive other humans? How important is it that

31 they look or sound human or behave like humans? What are our expectations from robots? To

what extent do they fulfill our expectations? These are some of the questions cognitive scientists are interested in addressing not only to be able to better understand human nature but also to be able to guide the design of robots in the future.

In his classical work, The Design of Everyday Things, Don Norman (2013) provides

important insights about how cognitive sciences can help in the design of artefacts including machines such as robots. According to Norman (2013), the design artifacts should be adapted to the minds of their users and this is why one needs to understand the human mind first. This implies that a collaboration between human-robot interaction and cognitive sciences is necessary. Indeed, the use of robots in well-established cognitive psychology and neuroscience paradigms in the last decade has proven useful to understand how humans respond to non-human agents

42 as compared to their human counterparts, and what kind of principles we should follow in 43 humanoid robot design (MacDorman and Ishiguro, 2006; Cross et al., 2019; Cross and Ramsey, 44 2020).

One of the cognitive psychology/neuroscience paradigms that have been successfully applied in human-robot interaction is expectation-violation paradigms (Saygin et al., 2012; Urgen

47 et al., 2018). These paradigms have been developed to understand the nature of information 48 processing in a variety of perceptual and cognitive tasks (Kutas and Hillyard, 1980; Kutas and 49 Federmeier, 2011; Kok et al., 2013; Kok and de Lange, 2015; de Lange et al., 2018; Urgen and 
51 cognition such as predictive coding or computation (Friston, 2010; Clark, 2013, Heeger, 2017).

52 According to these theories, perception is not a purely bottom-up or stimulus-driven process, 53 rather, expectations and prior knowledge play an important role in how we perceive our 54 environment. A growing body of empirical work in psychology and neuroscience are in line with 55 these theories showing that participants respond faster and more accurately when they perceive 56 events that are expected compared to the ones that are unexpected (Kok et al., 2013; Kok and 57 de Lange, 2015; de Lange et al., 2018). These results suggest that humans constantly predict 58 what would come next and this in turn determines what they perceive.

59 Recent work at the intersection of cognitive science and social robotics has shown that 60 humans can extend their prediction skills to perception of robots and form expectations about 61 robots based on their prior experience (Ho and MacDorman, 2008; Saygin et al., 2012; Urgen et 62 al., 2018). These studies manipulated expectations towards robots by means of using stimuli that 63 have mismatches in a variety of visual dimensions including appearance (form), motion, and 64 interaction. In other words, these mismatch paradigms aim to induce certain expectations based 65 on a particular cue, and at the same time present another cue that usually does not match with 66 that cue, resulting in expectation violation. For instance, Urgen et al. (2018) show that the 67 appearance of a robot can elicit certain expectations in humans about how the robot would move, 68 and when the robot does not move in the expected way, an N400 effect is observed indicating 69 that the expectations are violated.

70 Other studies explored mismatches in multisensory context including the congruity of 71 appearance and voice (Mitchell et al., 2011; Hastie et al., 2017; Cabral et al., 2017; Stein and 72 Ohler, 2018; McGinn and Torre, 2019). One drawback of these studies is that they usually use 73 subjective measures such as fear and eeriness (Mitchell et al., 2011), credibility or attractiveness

74 (Stein and Ohler, 2018), politeness and lifelikeness (Hastie et al., 2017), likability, expressiveness, 75 and understandability (Cabral et al., 2017), drawings (Mara et al., 2020), or emotion labeling 76 (Tsiourti et al., 2019) to evaluate artificial agents rather than more objective measures such as 
77 reaction time or accuracy, which would be more informative about the basic perceptual processes.

78 Hence, how expectations affect perception of robots in a cross-modal audio-visual context remain 79 unknown.

80 The aim of the present study is to investigate whether we make predictions about how

81 robots sound based on how they look, and whether those predictions are similar to the ones we

82 make for humans. To this end, we used an expectation-violation paradigm in which human

83 participants judged whether a greeting word sounded human-like or robotic. This sound was

84 preceded by a picture of a human or a robot and informed the participants with a certain probability

85 about the sound that would follow (thus form expectations). The hypothesis is that people would

86 perceive robot sounds faster when they are preceded by a robot picture in contrast to a human

87 picture just as they would do so with human sounds that are preceded by human pictures. Our

88 results confirm this hypothesis suggesting that we could develop certain expectations about how

89 robots behave (e.g. sound like) based on their appearance, similar to our expectations about our

90 human companions.

\section{Method}

\section{Participants}

9330 healthy adults from Bilkent University ( 16 females, Mean age $=25.2, \mathrm{SD}=.65$ ) participated in

94 the experiment. All participants had normal or corrected-to-normal vision and hearing. The sample

95 size of the study was determined by a power analysis prior to data collection. The minimum

96 required sample size was determined to be 30 by using $G^{*}$ Power (with alpha $=0.05$, beta $=0.90$,

$97 \eta 2=0.25)$. Before the experiment, all subjects signed a consent form approved by the Human

98 Research Ethics Committee of Bilkent University. 


\section{Stimuli}

102 Visual Stimuli: The visual stimuli consisted of static images of three agents. We call them Human, Android, and Robot (See Figure 1). Android and Robot are the same machine in different appearances. Android has a more human-like appearance, and was modeled from the Human agent, whereas Robot has a more mechanical appearance as the clothing is removed. Android is the robot Repliee Q2 which was developed at Osaka University. The images in Figure 1 were captured from the videos of Saygin-Ishiguro database (Saygin et al., 2012; Urgen et al., 2018),

108 the agents were doing hand waving gesture. The images were $240 \times 240$ pixels in size, and all 109 three were matched in terms of their low-level properties (luminance and spatial frequency) with 110 SHINE Toolbox (V. Willenbockel et al., 2010).

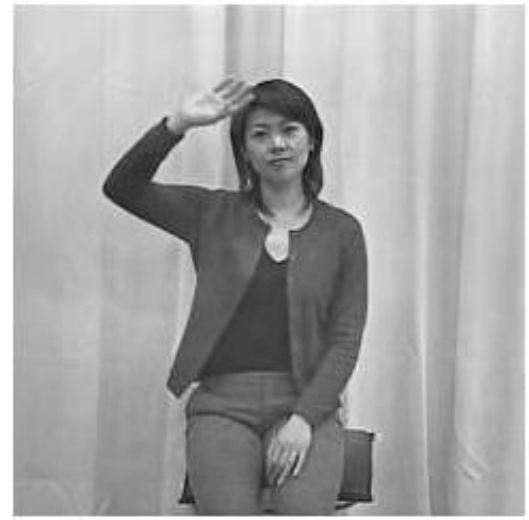

HUMAN

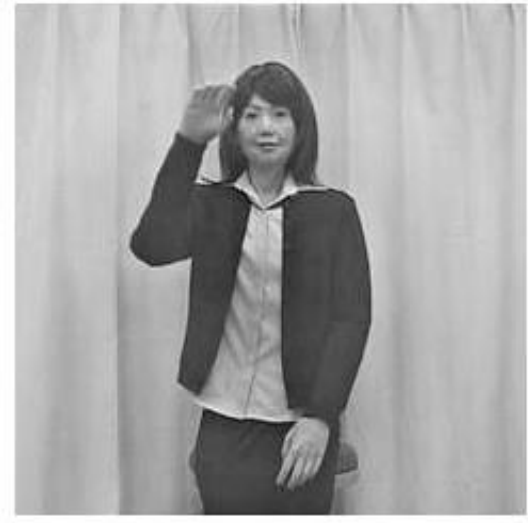

ANDROID

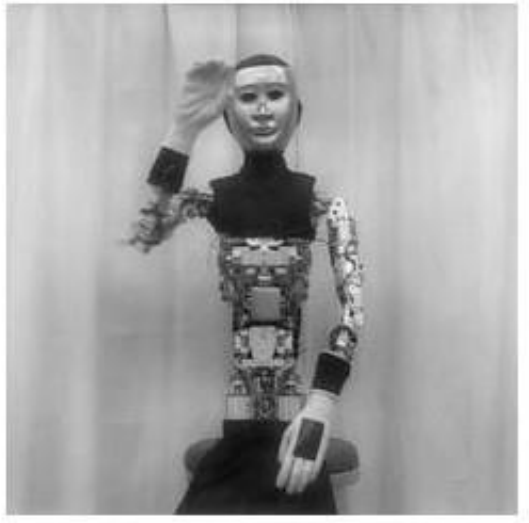

ROBOT

112 Figure 1. Visual stimuli in the experiments consist of images of three agents with different degrees of human-likeness: a human (Human), and two robots, one having more human-like appearance (Android) and one having less human-like appearance (Robot).

117 voice of a human saying 'Good morning' (Human Voice), and a modified version of it in which the

118 voice sounds robotic (Robotic Voice). To make the natural human voice robotic, we used an audio

119 library (AudioLib) in Python (Peirce, 2019). We used the settings of the "Ghost" option in this

120 library to create the robotic voice. To compensate for the echo factor in the robotic voice, echo

121 (0.05) was added to the human voice. Audio files were matched in terms of their amplitude using 
122 the Adobe Audition CC (13.0.6). We also added white noise to both sound files to make the task

123 harder, as previous research on prediction shows that the effect of prediction is strongest when

124 the stimulus is ambiguous (Lange et al., 2018).

\section{Procedure}

127 Subjects participated in two experiments (Experiment 1 and Experiment 2). The order of the 128 experiments was counterbalanced across subjects. In both experiments, the subjects were seated $12957 \mathrm{~cm}$ away from a computer screen. Their heads were stabilized with a chin-rest. Before each 130 experiment, the subjects were introduced to the visual and auditory stimuli, and were given verbal 131 instructions. In particular, the human voice was told to belong to the human in the Human image, 132 and the robotic voice was told to belong to the agent in the Robot or Android image depending 133 on the experiment. They also did a practice session to make sure that they understood the task. 134 The experiment was programmed in Psychtoolbox (Brainard, 1997; Pelli, 1997).

136 Experiment 1: Experiment 1 consisted of 5 blocks, each containing 80 trials. Each trial started 137 with a fixation cross on a gray background ( $1 \mathrm{sec}$ ), which was followed by a visual cue ( $1 \mathrm{sec})$, an 138 image of a human or a mechanical robot (Human and Robot agents in Figure 1). Following the 139 visual cue, a $2 \mathrm{sec}$ auditory stimulus was presented, either a human or a robotic voice (See Figure 140 2). The task of the subjects was to indicate whether the sound was human-like or robotic by 141 pressing a key. 


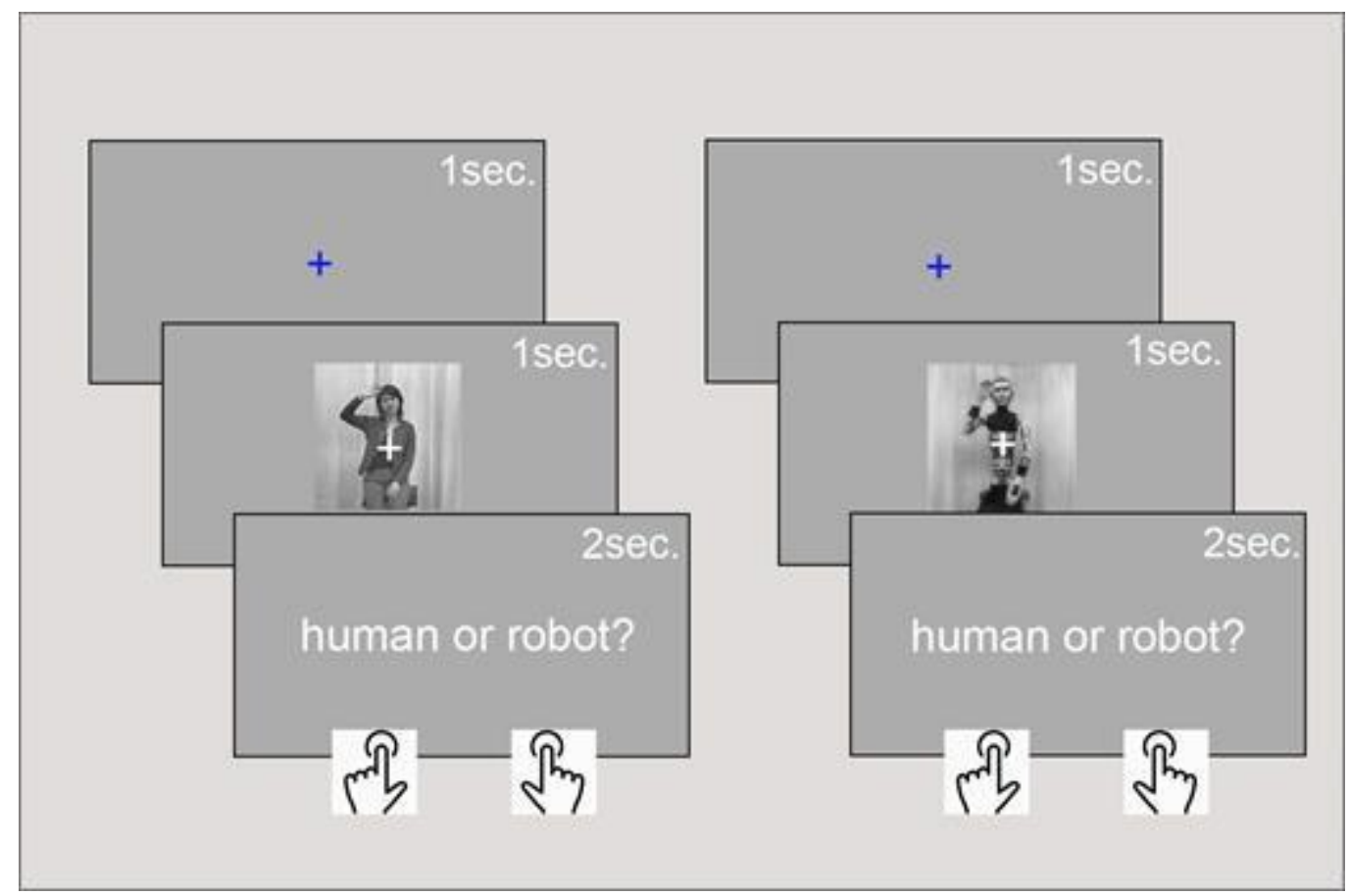

143 Figure 2. Each trial in Experiment 1 consists of a fixation screen, a visual cue (Human or Robot) and an 144 auditory target (human or robotic voice) after which subjects need to respond with a key press.

146 The visual cue informed the subjects about the upcoming auditory stimulus category. In $80 \%$

147 of the trials, the visual cue was congruent with the auditory stimulus (e.g. human image and 148 human voice or robot image and robotic voice), whereas in $20 \%$ of the trials, the visual cue was 149 incongruent with the auditory stimulus (e.g. human image and robotic sound or robot image and 150 human voice, see Figure 3). 


\section{A. CONGRUENT TRIALS}
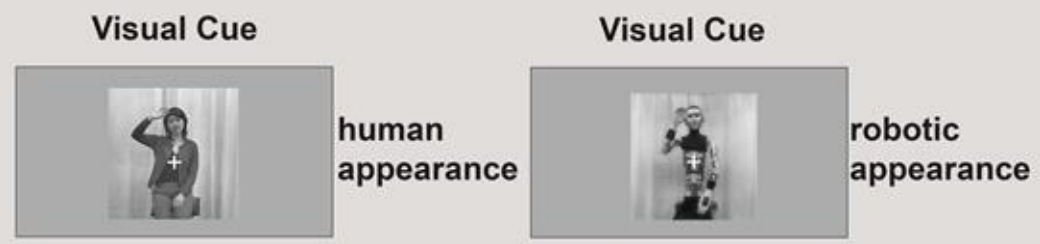

Target

(uf)) human voice

Target

(प्(j)) robotic voice
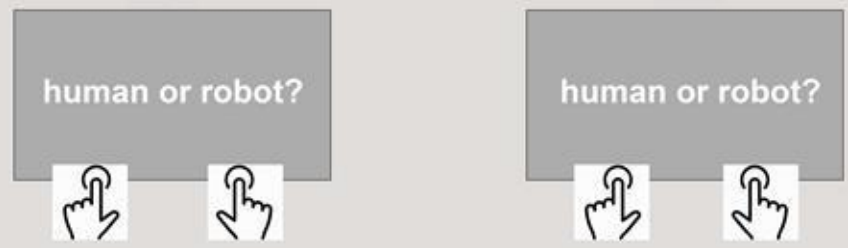

\section{B. INCONGRUENT TRIALS}
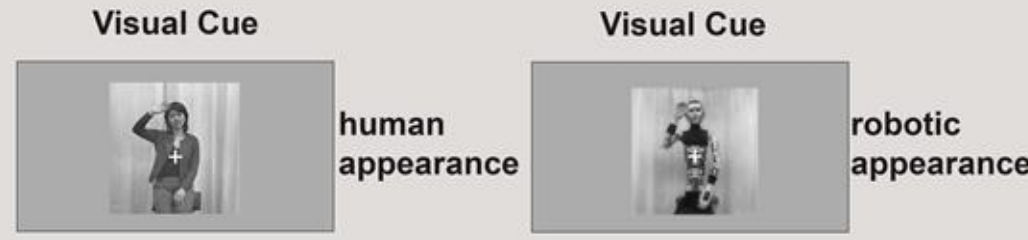

Target

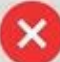

(40)) robotic voice
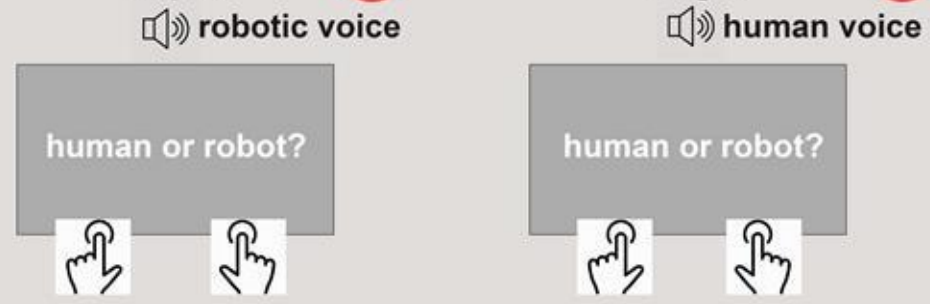

Figure 3. There are two types of trials in Experiment 1. A. Congruent trials in which the category of the visual cue and the auditory target match (e.g. human appearance (Human) and human voice, or robotic appearance (Robot) and robotic voice), B. Incongruent trials in which the category of the visual cue and the auditory target do not match (e.g. human appearance (Human) and robotic voice, or robotic appearance (Robot) and human voice).

158 Experiment 2: Experiment 2 is identical to Experiment 1 except the visual cue screen. As a visual cue, subjects were shown the image of either a human or a human-like robot (Human and Android agents in Figure 4). 


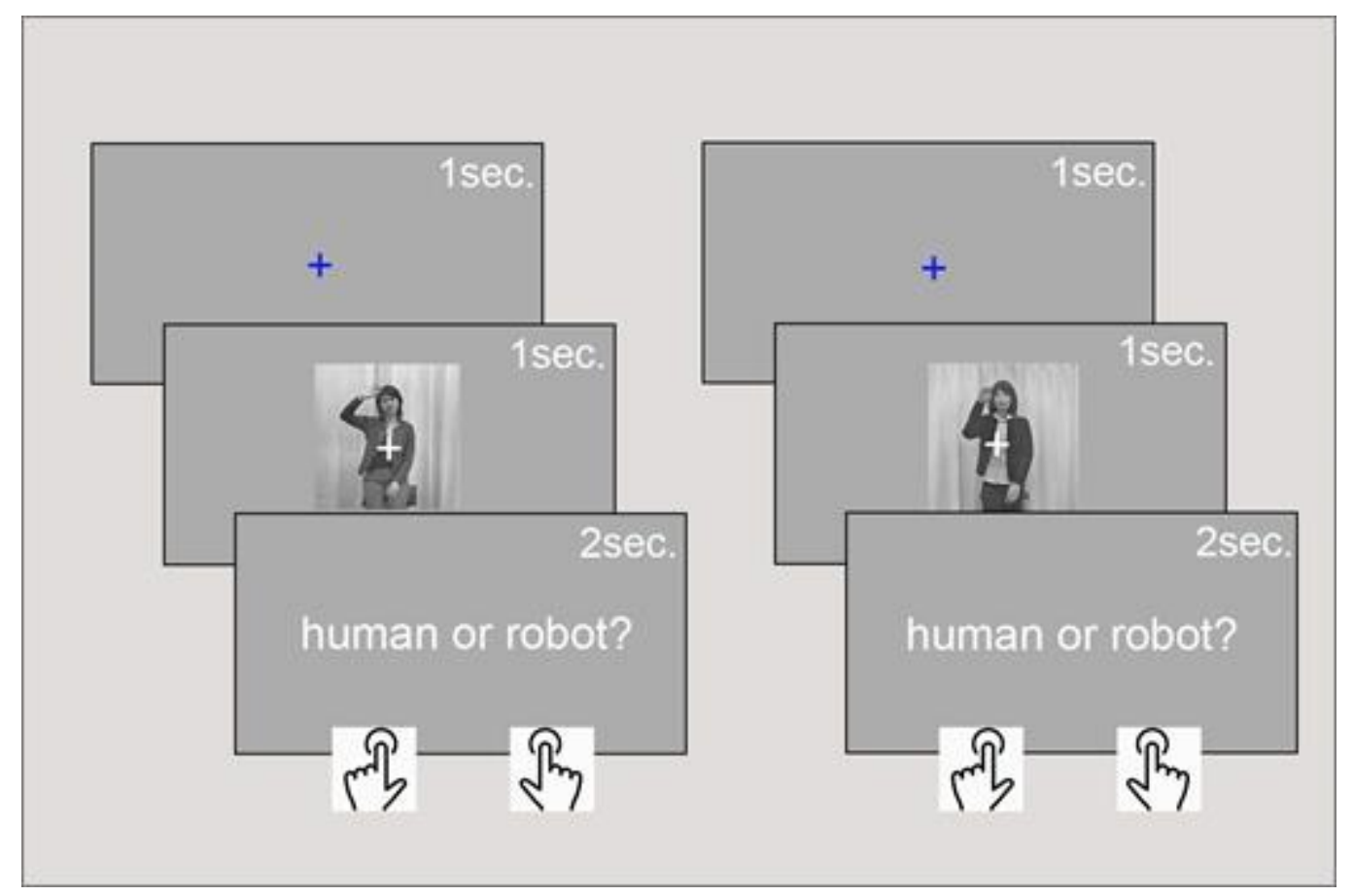

162 Figure 4. Each trial in Experiment 2 consists of a fixation screen, a visual cue (Human or Android) and an auditory target (human or robotic voice) after which subjects need to respond with a key press.

165 Similar to Experiment 1, there were two types of trials: congruent trials and incongruent trials. In 166 congruent trials ( $80 \%$ of total trials), the category of the visual cue matched the category of the 167 auditory stimulus (e.g. human image and human voice, or a robot image and robotic voice). In 168 incongruent trials, the category of the visual cue did not match the category of the auditory target 169 (e.g. human appearance and robotic voice, or robotic appearance and human voice, see Figure 170 5). The total number of trials was the same with Experiment 1. 


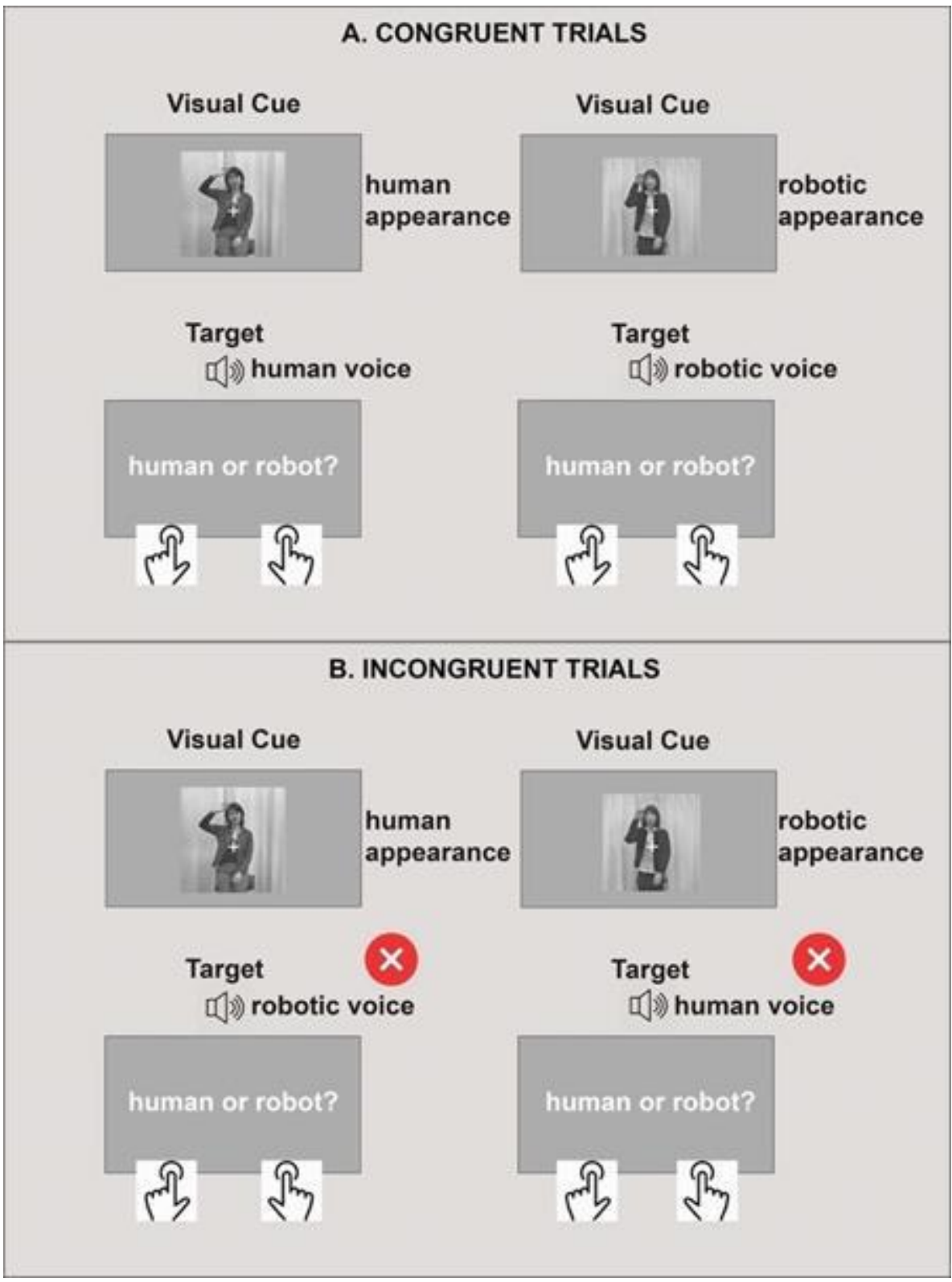

173 Figure 5. There are two types of trials in Experiment 2. A. Congruent trials in which the category of the visual cue and the auditory target match (e.g. human appearance (Human) and human voice, or robotic appearance (Android) and robotic voice), B. Incongruent trials in which the category of the visual cue and the auditory target do not match (e.g. human appearance (Human) and robotic voice, or robotic appearance

\section{Statistical Analysis}

180 We conducted separate ANOVAs for Experiment 1 and Experiment 2, and an additional ANOVA

181 to compare the results of Experiment 1 and Experiment 2. 
183 Experiment 1: We conducted 2 (Congruency: Congruent, Incongruent) x 2 (Visual Cue: Human,

184 Robot) repeated measures ANOVA to investigate the effects of congruency and visual cue (agent)

185 on reaction times and accuracy.

186 Experiment 2: We conducted 2 (Congruency: Congruent, Incongruent) x 2 (Visual Cue: Human, 187 Android) repeated measures ANOVA to investigate the effects of congruency and visual cue 188 (agent) on reaction times and accuracy.

189 Comparison of Experiment 1 and Experiment 2: We conducted 2 (Congruency: Congruent, 190 Incongruent) $\times 3$ (Visual Cue: Human, Android, Robot) to investigate whether the congrueny, the 191 human-likeness of the agent, or their interaction affect reaction times or accuracy.

\section{Results}

\section{Experiment 1 (Human, Robot)}

194 Reaction Times:

We found a main effect of congruency on reaction times $(F(1,29)=58.79, p<.05, \eta 2=$

.55). Subjects responded to auditory targets significantly faster in congruent trials (when the 197 category of the auditory target matched to the visual cue category) (Mean $=1.28 \mathrm{sec}, \mathrm{SD}=0.15$ ) 198 than incongruent trials (when the category of the auditory target did not match to the visual cue 199 category) (Mean $=1.47 \mathrm{sec}, \mathrm{SD}=0.16$ ). We also found a main effect of agent (visual cue) on 200 reaction times $(F(1,29)=10.48, p<.05, \eta 2=.04)$. Subjects were significantly faster when the visual 201 cue was Human (Mean $=1.35 \mathrm{sec}, \mathrm{SD}=0.03$ ) than it was Robot (Mean $=1.40 \mathrm{sec}, \mathrm{SD}=0.03$ ). 202 There was no interaction between the congruency and the visual cue $(F(1,29)=0.097, p=0.76)$. 203

We conducted pairwise t-tests between congruent and incongruent conditions for each 205 visual cue category (Human and Robot) separately. When the visual cue category was Robot, 
207 incongruent trials (Mean $=1.49 \mathrm{sec}, \mathrm{SD}=0.17)(\mathrm{t}(29)=-7.23, \mathrm{p}<.05)$. Similarly, when the visual 208 cue category was Human, subjects performed significantly faster in congruent (Mean $=1.26 \mathrm{sec}$, $209 \mathrm{SD}=0.14)$ than incongruent conditions (Mean $=1.44 \mathrm{sec}, \mathrm{SD}=0.16)(\mathrm{t}(29)-7.22, \mathrm{p}<.05)$.

210

\section{Accuracy:}

212 There was a main effect of congruency on accuracy scores $(F(1,29)=63.48, p<.001, \eta 2$ $213=.68)$. Subjects responded to auditory targets significantly more accurately in congruent trials 214 (when the category of the auditory target matched to the visual cue category) (Mean $=88.06 \%$, $215 S D=10.60$ ) than incongruent trials (when the category of the auditory target did not match to the 216 visual cue category) (Mean $=71.04 \%, S D=16.47)$. However, there was no significant effect of 217 visual cue on accuracy $(F(1,29)=0.082, p=0.78)$. We did not find an interaction between 218 congruency and visual cue, either $(F(1,29)=0.22, p=0.64)$.

We conducted pairwise t-tests between congruent and incongruent conditions for each 220 visual cue category (Human and Robot) separately for accuracy scores. Participants performed 221 significantly more accurately in congruent trials (Mean $=88.54 \%, S D=10.68$ ) than incongruent 222 trials $($ Mean $=71.08 \%, \mathrm{SD}=17.24)(\mathrm{t}(29)=7.40, \mathrm{p}<.05)$ when the visual cue was Human. We 223 also found that when the visual cue was Robot, subjects performed significantly more accurate in 224 congruent trials (Mean $=87.58 \%, S D=11.78)$ than incongruent trials $($ Mean $=71 \%, S D=18.50)$ $225(\mathrm{t}(29)=7.22, \mathrm{p}<.05)$. 

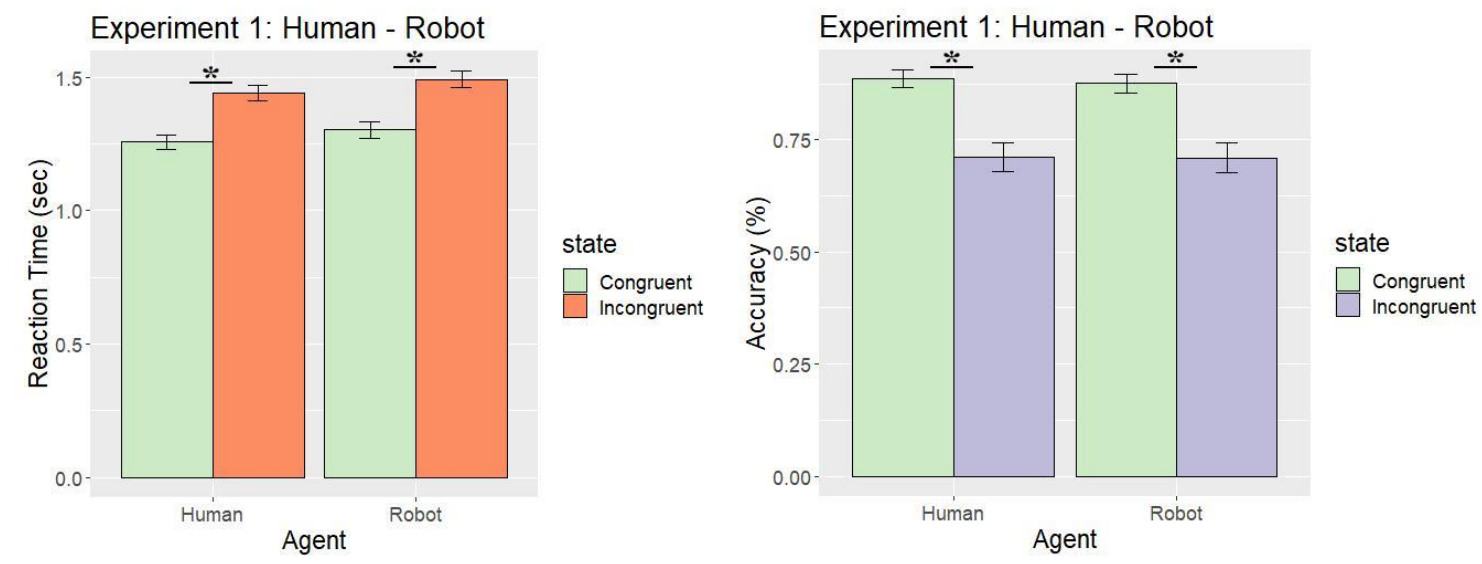

Figure 6. Reaction Times (RT) and Accuracy (\%) results of Experiment 1. Error bars show the standard error of the mean (SEM).

\section{Experiment 2 (Human, Android)}

231 Reaction Times:

We found a main effect of congruency on reaction times $(F(1,29)=54.65, p<.001, \eta 2$

$233=0.56$ ). Subjects responded to auditory targets significantly faster in congruent trials (when the 234 category of the auditory target matched to the visual cue category) (Mean $=1.27 \mathrm{sec}, \mathrm{SD}=0.16$ ) 235 than incongruent trials (when the category of the auditory target did not match to the visual cue 236 category) (Mean $=1.47 \mathrm{sec}, \mathrm{SD}=0.16$ ). We also found a main effect of visual cue on reaction 237 times $(F(1,29)=38.18, p<.001, \eta 2=0.04)$. Subjects were significantly faster when the visual cue 238 was Human (Mean $=1.34 \mathrm{sec}, \mathrm{SD}=0.03$ ) than it was Android (Mean $=1.40 \mathrm{sec}, \mathrm{SD}=0.03$ ).

239 There was no interaction between the congruency and the visual cue $(F(1,29)=0.90, p=0.35)$.

240 We conducted pairwise t-tests between congruent and incongruent conditions for each

241 visual cue category (Human and Android) separately. When the visual cue category was Android, 242 subjects performed significantly faster in congruent trials (Mean $=1.30 \mathrm{sec}, \mathrm{SD}=0.17$ ) than 243 incongruent trials (Mean $=1.50 \mathrm{sec}, \mathrm{SD}=0.16)(\mathrm{t}(29)=-6.94, \mathrm{p}<.05)$. Similarly, when the visual 
244 cue category was Human, subjects performed significantly faster in congruent (Mean = $1.26 \mathrm{sec}$,

$245 \mathrm{SD}=0.16)$ than incongruent conditions (Mean $=1.43 \mathrm{sec}, \mathrm{SD}=0.18)(\mathrm{t}(29)=-6.49, \mathrm{p}<.05)$.

247 Accuracy:

248 There was a main effect of congruency on accuracy scores $(F(1,29)=42.54, p<.001, \eta 2$

$249=.60)$. Subjects responded to auditory targets significantly more accurately in congruent trials

250 (Mean $=90.23, \mathrm{SD}=8.19)$ than incongruent trials (Mean $=71.29, \mathrm{SD}=20.58)$. However, there

251 was no effect of visual cue on accuracy $(F(1,29)=0.30, p=0.59)$. We also did not find an

252 interaction between congruency and visual cue $(F(1,29)=0.06, p=0.81)$.

253 We conducted pairwise t-tests between congruent and incongruent conditions for each

254 visual cue category (Human and Android) separately for accuracy scores. Participants performed

255 significantly more accurately in congruent trials (Mean $=90.52 \%, S D=8.11$ ) than incongruent 256 trials $($ Mean $=71.92 \%, \mathrm{SD}=21.03)(\mathrm{t}(29)=6.00, \mathrm{p}<.05)$ when the visual cue was Human. We

257 also found that when the visual cue was Android, subjects performed significantly more accurate 258 in congruent trials (Mean $=89.94 \%, S D=9.12)$ than incongruent trials $($ Mean $=70.83, S D=$ $25922.33)(t(29)=6.29, p<.05)$.
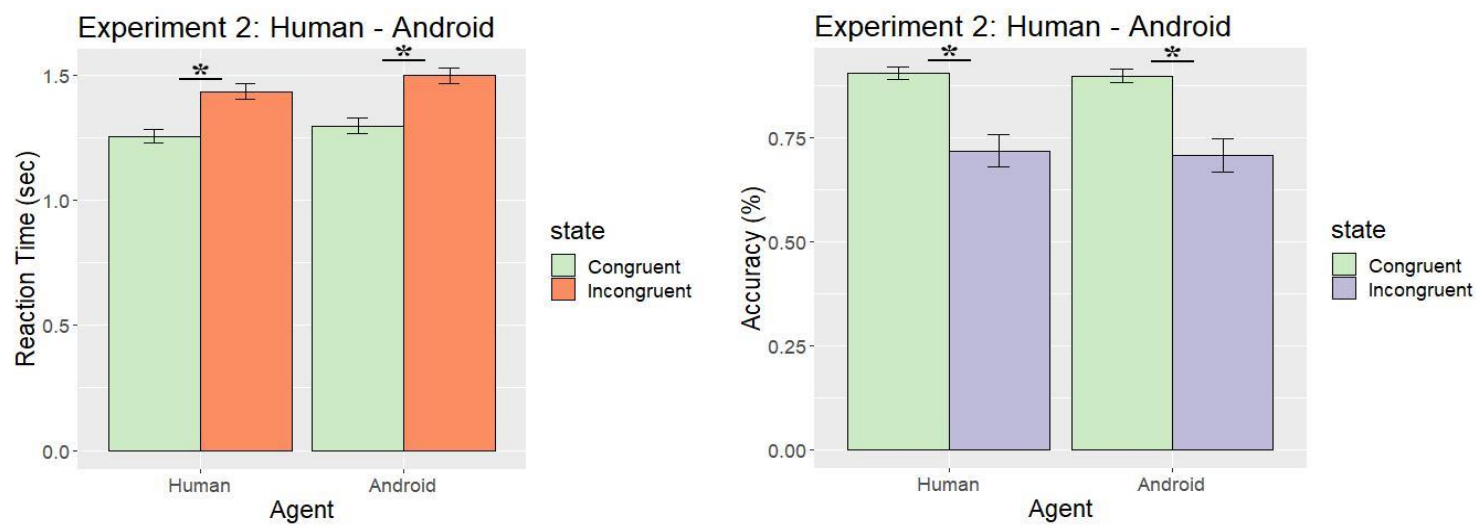

Figure 7. Reaction Times (RT) and Accuracy (\%) results of Experiment 2. Error bars show the standard 262 error of the mean (SEM). 

of the agent in the spectrum of Human-Android-Robot affected the reaction times or accuracy. To this end, we compared the reaction times of Experiment 1 and Experiment 2. Since the Human 267 agent existed in both experiments, we did two separate ANOVAs.

\section{Reaction Times:}

First we ran a 2 (Congruency: Congruent, Incongruent) x 3 (Visual Cue: Human, Android, 270 Robot) ANOVA using the Human and Robot RTs from Experiment 1, and Android RTs from 271 Experiment 2. We found a main effect of congruency $(F(1,29)=68.98, p<.05, \eta 2=0.50)$ and of 272 visual cue $(F(1,29)=4.26, p<.05, \eta 2=0.03)$. Pair-wise comparisons (Bonferroni-corrected) 273 between the three agents (visual cues) show that participants were faster when the visual cue 274 was Human as compared to Android ( $\mathrm{t}=-2.512, \mathrm{p}=0.044)$ or Robot $(\mathrm{t}=-2.548, \mathrm{p}<.05)$. They 275 responded similarly when the visual cue was Robot or Android ( $t=-0.036, p=1.0)$. There was no interaction between congruency and visual cue $(F(1,29=0.26, p=.07)$.

We ran a second 2 (Congruency: Congruent, Incongruent) $\times 3$ (Agent: Human, Android, 278 Robot) ANOVA using the Human and Android RTs from Experiment 2, and Robot RTs from 279 Experiment 1. We found a main effect of congruency $(F(1,29)=67.86, p<.05, \eta 2=0.47)$, and of 280 visual cue $(F(1,29)=4.36, p<.05, \eta 2=0.03)$. There was not an interaction between congruency 281 and visual cue $(F(1,29=0.36, p=.70)$. Pairwise comparisons (Bonferroni-corrected) between the 282 three agents (visual cues) show that participants were faster when the visual cue was Human as 283 compared to Android $(t=-2.540, p<.05)$ or Robot $(t=-2.573, p<.05)$. They responded similarly 284 when the visual cue was Robot or Android $(t=-0.033, p=1.0)$.

286 Accuracy:

287 For completeness, we also ran two 2 (Congruency: Congruent, Incongruent) x 3 (Agent: 288 Human, Android, Robot) ANOVAs combining the scores of Experiment 1 and Experiment 2. In 
289 the first ANOVA, we combined the Human and Robot scores in Experiment 1 and Android scores

290 in Experiment 2. We found a main effect of congruency $(F(1,29)=70.44, p<.05, \eta 2=0.65)$.

291 However, there was no significant effect of the visual cue $(F(1,29=0.19, p=0.83)$. We also did

292 not find an interaction between congruency and visual cue $(F(1,29=0.49, p=0.62)$.

293 In the second ANOVA, we combined the Human and Android accuracy scores in

294 Experiment 2 and Robot scores in Experiment 1. Our results show that there was a main effect 295 of congruency $(F(1,29)=54.52, p<.05, \eta 2=0.65)$. However, we did not have a significant effect 296 of visual cue $(F(1,29)=0.66, p=0.52)$. We also did not find an interaction between congruency 297 and visual cue $(F(1,29=0.59, p=0.56)$.

\section{Discussion}

We investigated whether expectations about artificial agents affect our perception. To this end, we used a well-known prediction paradigm from cognitive psychology in human-robot interaction context. We hypothesized that people would get faster in judging how an agent sounds like (human-like or robotic) if it was preceded by a congruent visual cue (e.g. a robotic picture for 304 a robotic voice) than an incongruent visual cue (e.g. a human-like picture for a robotic voice).

Our results suggest that people form expectations about how an agent sounds based on

306 the appearance of the agent. For instance, if the visual cue is a robot, people expect that it would 307 sound robotic, as demonstrated by shorter reaction times and more accurate responses when the 308 appearance and voice were congruent than when they were incongruent. This was true whether 309 the robot has a more human-like appearance or less human-like. These results are consistent 310 with previous work that suggests that predictive processes underlie our perception (Friston, 2010; 311 Clark, 2013; Kok et al., 2013; Kok and de Lange, 2015; de Lange et al., 2018) including humans 312 and robots. In other words, it seems that we can extend our predictive capabilities to perceive 313 artificial agents, and just like our interaction with other humans, our expectations can affect how 314 we perceive non-human agents. 
Although there have been studies that examined the congruence of appearance and voice

316 in perception of robots, most of these studies use subjective measures in characterizing people's

317 experience with these agents (Mitchell et al., 2011; Hastie et al., 2017; Cabral et al., 2017; Stein

318 and Ohler, 2018; Tsiourti et al., 2019; Mara et al., 2020). We extend this work by characterizing

319 perception with more objective measures such as reaction time and accuracy. One advantage of

320 this approach is that it has enabled us to make more direct links with the perception literature in

321 cognitive sciences. Consistent with previous work on predictive processing in perception of simple

322 or complex object stimuli (Kok et al., 2013; Kok and de Lange, 2015; de Lange et al., 2018), we

323 found that reaction times get longer, and accuracy scores get lower when we encounter stimuli

324 that we do not expect. This in turn suggests that our expectations affect how we perceive non-

325 human agents as they do with other object categories.

What is the importance of studying how our expectations affect our perception of robots practical implications? Previous work suggests that an important criterion in the design of robots is to avoid the uncanny valley (Mori, 1970). Uncanny valley is characterized as a non-linear relationship between the degree of human-likeness an artificial agent possesses and humans' reaction to it: It has been shown that people show increasingly positive responses as an artificial agent gets more human-like up to a point where the resemblance of a real human induces completely opposite, negative responses (MacDorman et al., 2009). One prominent explanation

334 for the uncanny valley is that artificial agents which are almost human-like lead people to think 335 that they possess human norms, so they expect them to look and act completely human, but 336 because of their imperfections in one way or the other, they violate people's expectations (Saygin 337 et al., 2012; Urgen et al., 2018). The implication of this explanation is that roboticists should design 338 artificial agents in such a way that they do not violate people's expectations, because otherwise 339 people may find them eerie and may not want to interact with them (Yamamoto et al., 2009; 340 Cheetham et al., 2013; Mitchell et al., 2012; Tinwell et al., 2010; 2015; MacDorman and 
341 Chattopadhyay, 2016; Lee, 2010). So, in this context, what we expect from robots and in what 342 ways they may violate our expectations become an important research question. Appearance and 343 voice examined in the present study as well as in some other work (Mitchell et al., 2011; Tinwell 344 et al., 2015) are only two features among many, for which we seek a match in agent perception.

345 Future work could investigate what features of artificial agents make us form expectations, how 346 we do that, and under what conditions these expectations are violated.

347 Another implication of the present study for future research is about individual differences 348 in perception of robots. An interesting finding of our study is that people are overall faster when 349 the visual cue that triggers expectations is a human than a robot, regardless of how human-like 350 the robot is. One potential explanation of this finding is that people are much more familiar with 351 other humans than robots, and this familiarity might have facilitated how expectations influence 352 perception. On the other hand, recent research suggests that people may differ in how they 353 respond to artificial agents (Macdorman and Entezari, 2015), consistent with other work in 354 cognitive sciences (Kanai and Rees, 2011). One important question in the context of the present 355 study is whether there are individual differences in forming expectations about robots. Note that expectations are formed based on previous experiences. Thus, one could predict that familiarity 357 with robots (how much you know about them) can affect what we expect from them. For instance, 358 an engineer in Japan who is heavily exposed to robots may not be surprised by a metallic-looking 359 robot speaking with a humanlike voice as much as a person who has never interacted with a 360 robot. Similar concerns may apply when we consider different generations. For instance, children 361 who are born in the last decade in the technology era may have different expectations from robots 362 compared to elderly. Future work should investigate how familiarity with robots can affect our 363 prediction abilities. This will enable the design of customized robots for different end users.

364 The present study also sets as a good example about how the collaboration between 365 human-robot interaction and cognitive sciences can be fruitful and useful for both sides. Our study 366 not only suggests possible principles for robot design but also shows how fundamental cognitive 
367 mechanisms such as prediction can generalize to agents that we have not evolved with over many 368 generations. As such, our study shows that artificial agents such as robots can be great 369 experimental tools for cognitive science to improve our understanding of the human mind.

\section{References}

372 Brainard, D. H., \& Vision, S. (1997). The psychophysics toolbox. Spatial vision, 10(4), 433-436.

374 Cabral, J. P., Cowan, B. R., Zibrek, K., \& McDonnell, R. (2017). The Influence of Synthetic Voice 375 on the Evaluation of a Virtual Character. In INTERSPEECH (pp. 229-233).

377 Cheetham, M., Pavlovic, I., Jordan, N., Suter, P., \& Jancke, L. (2013). Category processing and 378 the human likeness dimension of the uncanny valley hypothesis: eye-tracking data. Frontiers in 379 psychology, 4, 108.

Clark, A. (2013). Whatever next? Predictive brains, situated agents, and the future of cognitive 382 science. Behavioral and brain sciences, 36(3), 181-204.

384 Cross, E. S., \& Ramsey, R. (2020). Mind Meets Machine: Towards a Cognitive Science of 385 Human-Machine Interactions. Trends in Cognitive Sciences.

387 Cross, E. S., Hortensius, R., \& Wykowska, A. (2019). From social brains to social robots: applying 388 neurocognitive insights to human-robot interaction.

390 De Lange, F. P., Heilbron, M., \& Kok, P. (2018). How do expectations shape perception?. Trends 391 in cognitive sciences, 22(9), 764-779. 
393 De Loof, E., Van Opstal, F., \& Verguts, T. (2016). Predictive information speeds up visual 394 awareness in an individuation task by modulating threshold setting, not processing efficiency.

395 Vision research, 121, 104-112.

396

397 Doehrmann, O., \& Naumer, M. J. (2008). Semantics and the multisensory brain: how meaning 398 modulates processes of audio-visual integration. Brain research, 1242, 136-150.

Friston, K. (2010). The free-energy principle: a unified brain theory?. Nature reviews 401 neuroscience, 11(2), 127-138.

402

403 Hastie, H., Lohan, K., Deshmukh, A., Broz, F., \& Aylett, R. (2017, November). The interaction 404 between voice and appearance in the embodiment of a robot tutor. In International Conference 405 on Social Robotics (pp. 64-74). Springer, Cham.

406

407 Hein, G., Doehrmann, O., Müller, N. G., Kaiser, J., Muckli, L., \& Naumer, M. J. (2007). Object 408 familiarity and semantic congruency modulate responses in cortical audiovisual integration areas. 409 Journal of Neuroscience, 27(30), 7881-7887.

411 Ho, C. C., MacDorman, K. F., \& Pramono, Z. D. (2008, March). Human emotion and the uncanny 412 valley: a GLM, MDS, and Isomap analysis of robot video ratings. In 2008 3rd ACM/IEEE 413 International Conference on Human-Robot Interaction (HRI) (pp. 169-176).

415 Ishiguro, H. (2006). The Uncanny Advantage of Using Androids in Social and Cognitive Science 416 Resarch. Interaction Studies, 7(3). 
418 Kanai, R., \& Rees, G. (2011). The structural basis of inter-individual differences in human 419 behaviour and cognition. Nature Reviews Neuroscience, 12(4), 231-242.

420

421 Kok, P., \& de Lange, F. P. (2015). Predictive coding in sensory cortex. In An introduction to model422 based cognitive neuroscience (pp. 221-244). Springer, New York, NY.

424 Kok, P., Brouwer, G. J., van Gerven, M. A., \& de Lange, F. P. (2013). Prior expectations bias 425 sensory representations in visual cortex. Journal of Neuroscience, 33(41), 16275-16284.

427 Laurienti, P. J., Kraft, R. A., Maldjian, J. A., Burdette, J. H., \& Wallace, M. T. (2004). Semantic 428 congruence is a critical factor in multisensory behavioral performance. Experimental brain 429 research, 158(4), 405-414.

430

431 Lee, E. J. (2010). The more humanlike, the better? How speech type and users' cognitive style 432 affect social responses to computers. Computers in Human Behavior, 26(4), 665-672.

434 MacDorman, K. F., \& Chattopadhyay, D. (2016). Reducing consistency in human realism 435 increases the uncanny valley effect; increasing category uncertainty does not. Cognition, 146, $436 \quad 190-205$.

438 MacDorman, K. F., \& Entezari, S. O. (2015). Individual differences predict sensitivity to the 439 uncanny valley. Interaction Studies, 16(2), 141-172.

441 MacDorman, K. F., Green, R. D., Ho, C. C., \& Koch, C. T. (2009). Too real for comfort? Uncanny 442 responses to computer generated faces. Computers in human behavior, 25(3), 695-710. 
444 Mara, M., Schreibelmayr, S., \& Berger, F. (2020, March). Hearing a nose? User expectations of 445 robot appearance induced by different robot voices. In Companion of the 2020 ACM/IEEE 446 International Conference on Human-Robot Interaction (pp. 355-356).

448 McGinn, C., \& Torre, I. (2019, March). Can you tell the robot by the voice? an exploratory study 449 on the role of voice in the perception of robots. In 2019 14th ACM/IEEE International Conference 450 on Human-Robot Interaction (HRI) (pp. 211-221). IEEE.

452 Mitchell, W. J., Szerszen Sr, K. A., Lu, A. S., Schermerhorn, P. W., Scheutz, M., \& MacDorman, 453 K. F. (2011). A mismatch in the human realism of face and voice produces an uncanny valley. i454 Perception, 2(1), 10-12.

456 Mitchell, W.J., Szerszen, K.A., Lu, A.S., Schermerhorn, P.W., Scheutz, M. and MacDorman, K.F. 457 2011. A mismatch in the human realism of face and voice produces an uncanny valley. 458 Iperception, 2(1): 10-12.

459

460 Nie, J., Park, M., Marin, A.L., Sundar, S.S. 2012. Can you hold my hand? Physical warmth in 461 human-robot interaction. Human-Robot Interaction. Boston, Massachusetts, USA.

463 Norman, D. (2013). The design of everyday things: Revised and expanded edition. Basic books. 464

465 Pelli, D. G., \& Vision, S. (1997). The VideoToolbox software for visual psychophysics: 466 Transforming numbers into movies. Spatial vision, 10, 437-442. 
468 Saygin, A. P., Chaminade, T., Ishiguro, H., Driver, J., \& Frith, C. (2012). The thing that should not 469 be: predictive coding and the uncanny valley in perceiving human and humanoid robot actions. 470 Social cognitive and affective neuroscience, 7(4), 413-422.

471

472 Stein, J. P., \& Ohler, P. (2018). Uncanny... But Convincing? Inconsistency Between a Virtual 473 Agent's Facial Proportions and Vocal Realism Reduces Its Credibility and Attractiveness, but Not 474 Its Persuasive Success. Interacting with Computers, 30(6), 480-491.

476 Talsma, D. (2015). Predictive coding and multisensory integration: an attentional account of the 477 multisensory mind. Frontiers in Integrative Neuroscience, 9, 19.

479 Tinwell, A., Grimshaw, M., \& Nabi, D. A. (2015). The effect of onset asynchrony in audio-visual 480 speech and the Uncanny Valley in virtual characters. International Journal of Mechanisms and 481 Robotic Systems, 2(2), 97-110.

482

483 Tinwell, A., Grimshaw, M., \& Williams, A. (2010). Uncanny behaviour in survival horror games. 484 Journal of Gaming \& Virtual Worlds, 2(1), 3-25.

485

486 Tsiourti, C., Weiss, A., Wac, K., \& Vincze, M. (2019). Multimodal integration of emotional signals 487 from voice, body, and context: Effects of (in) congruence on emotion recognition and attitudes 488 towards robots. International Journal of Social Robotics, 11(4), 555-573.

489

490 Urgen, B. A., Kutas, M., \& Saygin, A. P. (2018). Uncanny valley as a window into predictive 491 processing in the social brain. Neuropsychologia, 114, 181-185. 
493 Urgen, B. M., \& Boyaci, H. (2019). When expectations are not met: unraveling the computational 494 mechanisms underlying the effect of expectation on perceptual thresholds. bioRxiv, 545244.

495

496 Willenbockel, V., Sadr, J., Fiset, D., Horne, G. O., Gosselin, F., \& Tanaka, J. W. (2010).

497 Controlling low-level image properties: the SHINE toolbox. Behavior research methods, 42(3),

$498 \quad 671-684$.

499

500 Yamamoto, K., Tanaka, S., Kobayashi, H., Kozima, H., \& Hashiya, K. (2009). A non-humanoid

501 robot in the "uncanny valley": experimental analysis of the reaction to behavioral contingency in

502 2-3 year old children. PloS one, 4(9), e6974.

503 